\title{
Social Context Representation in Product-Service Systems with Internet of Things
}

\author{
Yongse Kim, Kumiko Suzuki \\ Service Design Institute, Sungkyunkwan University, Suwon, Korea \\ Email:yskim@skku.edu
}

Received 13 June 2015; accepted 7 July 2015; published 14 July 2015

\begin{abstract}
A Product-Service Systems (PSS) is composed of service and product element to provide values for relevant stakeholders. A typical service interaction involves providers and receivers, and respective values are provided in a social interaction. When service channel is supported by physical touchpoints, receivers will interact with products, which may interact with providers. Thus, in PSS, various kinds of interactions occur in diverse social contexts. These social contexts can be represented using graphs where nodes represent stakeholders and product touchpoints and attributed edges represent the interaction. Interaction among products as well as that of human stakeholders and that of human and product touchpoint can be described using such a graph-based representation. In designing and operating PSS with internet of things, social context representations could be used in analyzing and comparing key aspects of social interaction types to retrieve similar cases to exploit related interaction design strategies and operation know-hows.
\end{abstract}

\section{Keywords}

Product-Service Systems, Service Design, Internet of Things, Social Context, Service Innovation

\section{Introduction}

Product-Service Systems (PSS) composed of services and products tightly integrated as systems and business models have been recognized as the emerging concept to address diverse values. In PSS, the integrated design for people-centered value is the key issue because of the value creation paradigm [1]. Designing activities of various stakeholders considering interaction with other stakeholders as well as products is essential in designing PSS. According to Goedkoop et al. (1999), interaction between service provider and client is often seen as a key item of service [2]. Interactions between stakeholders and inter-actions with products can be designed addressing human activities composed of perception, judgment and action influenced by messages of the counterpart, either human or product respectively [3].

For product use, ISO 20282-1 [4] emphasized the importance of social environment of use as one of context aspects of use. Social contexts are even more crucial in service interactions because of service in performed by 
activities of providers and receivers in interactive manners. As the social context is an important issue in PSS, proper representations of social contexts for various kinds will be needed to compare various PSS concepts and support designing processes. This paper will describe a representation method of social context of service interaction in PSS with internet of things (IoT). As a specific case of PSS with IoT, Smart Plug PSS cases are used to demonstrate the validity and utility of the representation.

\section{Social Context Representation}

\subsection{Social Context}

ISO 20282-1 [4] referred that social factors such as interpersonal interaction and the desire for privacy should be taken into account, where appropriate, when a product is designed to be used by more than one person at a time or where the user is in a group situation. Based on this, we defined two kinds of social context: interpersonal interaction and the desire for privacy, and this paper focus on an interpersonal interaction of social context.

To represent a social interaction context of an activity in PSS, we attempt to describe three different entities of a service provider, a service receiver and a product touchpoint. These are represented as a node each. We define them as a provider node, a receiver node and a product touchpoint node to distinguish. We used a circle node as representing a service receiver, a triangle node as representing a service provider and a square node as representing a product touchpoint. The directed edge shows interaction between service providers, receivers and touchpoints. We describe the interaction among these nodes as directed edge. Thus the social context is shown as a form of a directed graph. Through this, we attempt to represent the social context systematically.

Exemplary cases of the social contexts of PSS can be found in a hospital service, urban umbrella PSS, a restaurant service. In Figure 1, the graph representation (A) is a case of more than one provider is interacting with a receiver. Hospital service is a good example for showing this graph representation. When patients represented by a circle node as a service receiver visit hospital and receive its services, they will get services from many kinds of service providers like doctors, nurses, or radiotherapists represented by a triangle node. These providers collectively represent hospital service providers, and this is reflected in the undirected edges between them. The graph representation (B) is a case with a physical touchpoint. When service channel is supported by physical touchpoints, receivers would interact with product, which may interact with providers. Urban umbrella rental PSS [5] shows that a citizen can rent an umbrella through the renting station while a provider stocks up umbrellas in the station. That is, a service receiver and a service provider can have interaction through the product touchpoint represented as a square node without actual facing each other. The graph representation (C) includes a self-service. The self-service restaurant represented by (C) is that customers can bring ingredients and cooking

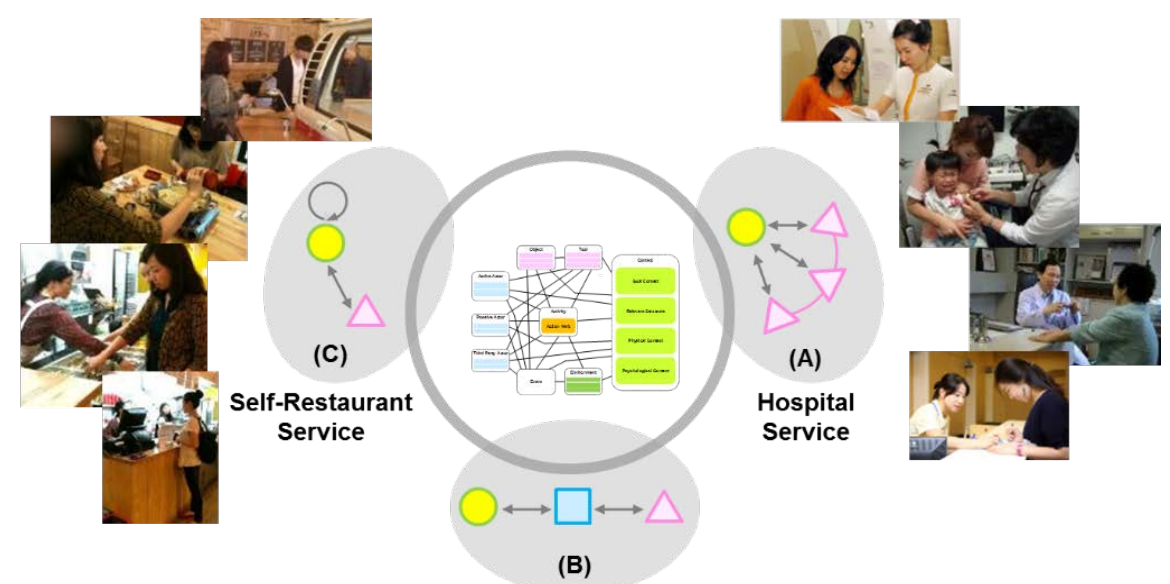

Urban Umbrella Rental Service System

Service Receiver

Service Provider

Product Touchpoint

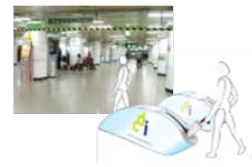

Figure 1. Social context representation. 
tools from the space restaurant arranged and cook by themselves. This self-restaurant service can be represented by the interaction between customer and restaurant staff, and also interaction for customers themselves while they provide some services for themselves. This self-service is characterized by a special edge with the same starting and ending nodes which, unlike other linear edges, represents a provider as a receiver.

For a directed graph, the direction of the edge, that is the interaction direction, can be either one way or two ways. While most of human-human interactions are two ways, interaction between human and product could be one directional with actions are concerned. However, note that these actions are due to perceptions and judgements of human, and perception is based on affordances given by products. Note also that the edges in a typical graph can contain more information as attributed edges. In this respect, the proposed simple representation of social context of this paper possesses potential to include much more diverse information through edge attributes.

\subsection{Case Example of Social Context}

We now describe the social contexts for example cases using the graph representation as the PSS concepts are developed: urban umbrella rental PSS and make-up room PSS.

Urban Umbrella Rental PSS. This PSS [5] has been designed exploiting the channel sharing strategy of channel aspect of the business model canvas, while extending the customer segment using metro stations as shared channel as given in Figure 2 [1]. In this PSS concept, the graph representation for social context-based interaction among a service provider, a service receiver and a product touchpoint would be as shown on the low-right of the rental PSS journey map in Figure 2. The user represented by the circle node can receive the umbrella through the rental service system represented as a square node, and the administrator as left triangle node manages and contacts with umbrella provider represented as right triangle node.

Make-Up Room PSS. A make-up room PSS [6] has been designed to allow customers to perform self-makeup in an open way with some payment. Figure 3 shows the scenario and the graph representation of social-context based interaction between stakeholders of make-up room service providing self-make-up. There comes the square node representing make-up room as a touchpoint, so service providers and receivers interact through the touchpoint by providing make-up room and paying membership fee, and service receivers perform the make-up service by themselves. This self-service is characterized by a special edge with cycling shape which shows that a provider and a receiver are the same.

\section{Case of a Product-Service System with Internet of Things: Smart Plug Product-Service System}

Experience and service design for smart plug product-service systems has been con-ducted to enable energy saving and enjoyable use of high-energy electric devices. A smart plug is a new device that measures electricity use through each plug outlet with on/off control [7] [8]. Users are enabled to use electrical devices in their specific customized manner with detailed energy consumption information through smart plug PSS Plug [9]. A service scenario with an electric rice cooker is reviewed and the social context of the service interaction of the corresponding PSS is described using the pro-posed graph representation. Social context representation of another

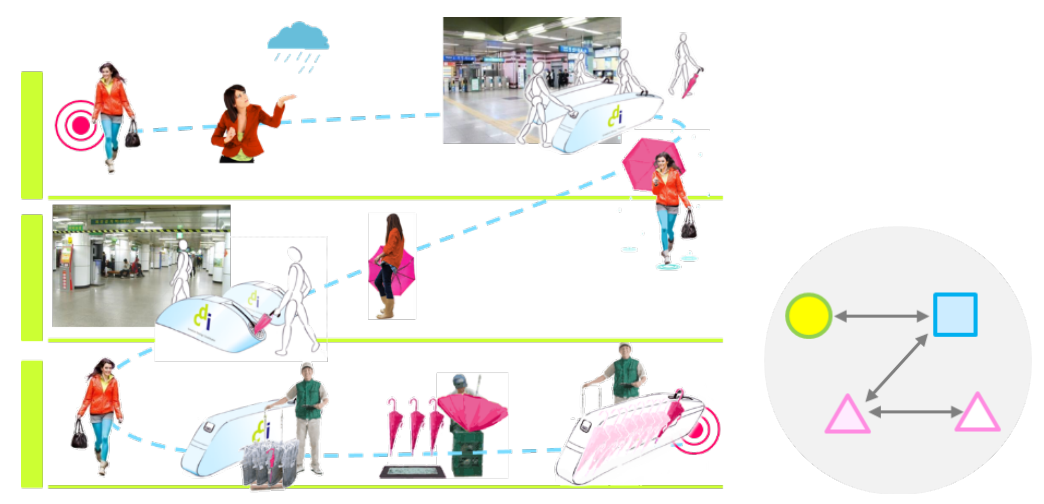

Figure 2. Scenario of urban umbrella rental PSS. 




Figure 3. Scenario of make-up room pss providing self-make-up.

PSS is shown. Then the two representations are merged to represent a typical use case of smart plugs with some electric devices where electric device usage and corresponding energy consumption are managed as a case of PSS with IoT.

\subsection{Service Journey Map \& Service Model}

A mother of 4-person family reviewed the electric bill of the last month. With a family meeting, she share with the members of her family on savings guide. They assign responsible person for each device and make a family-wide effort in saving energy. She is in charge of electric devices in the kitchen and she decides to use Smart Plug in conjunction with a rice cooker, which is a high energy device. First, she downloads the smart plug app and input rice cooker as a device used. As she uses different rice cooking functions, she records the amounts of energy usage with specific rice cooking function modes used. Through comparative evaluation of the energy uses and rice cooking function modes, she stores her customized modes. The service journey map of using an electric rice cooker is illustrated in Figure 4.

The service model of the smart plug PSS with a rice cooker is shown in the form of a customer value chain [10] in Figure 5. Smart plug app and rice cooker app are connected so that these apps communicate and share information. It provides value through service based on the interation with the user and the rice cooker app. Note that smart plug app must be connected with many appliance apps.

\subsection{Social Context Representation}

The user cooks rice using the rice cooker provided by the rice cooker company with the smart plug connected. The user of the rice cooker is also a user of the smart plug. These interactions are represented in the center part of Figure 6 with the user node connected to the rice cooker and the smart plug. Also the rice cooker and the smart plug are connected in Figure 6.

The user inputs the rice cooking modes in the rice cooker app and state information of the rice cooker is sent to the rice cooker app. The energy usage information is sent to the rice cooker app from the spart plug app, which is connected with the smart plug. The user may also get some information from the smart plug app directly. The rice cooker company provides both the rice cooker and its app. The same is true for the smart plug. This service is enabled by the interactions among human stakeholders and product touchpoints connected through internet, that is, internet of things.

For another electric device, an electric stove connected with a smart plug is considered. The user manages her usage information such as cooking recipe and corresponding stove strength and heating time on the electric stove app connected with the stove. The energy usage information is provided by the smart plug app. The interactions among its user, the electric stove, and the smart plug are represented in Figure 7 in a horizontally symmetric way with respect to those of the rice cooker.

As the user is using both the rice cooker and the electric stove, the user nodes are identified to be merged as shown in Figure 8. While two smart plugs are used for the rice cooker and the electric stove respectively, their apps would be identified as a common app. Also the nodes of the smart plug company would be merged. Thus the social context of the service interactions of the smart plug PSS with connection of the rice cooker, the electric stove, and the smart plugs would be represented in Figure 8. 


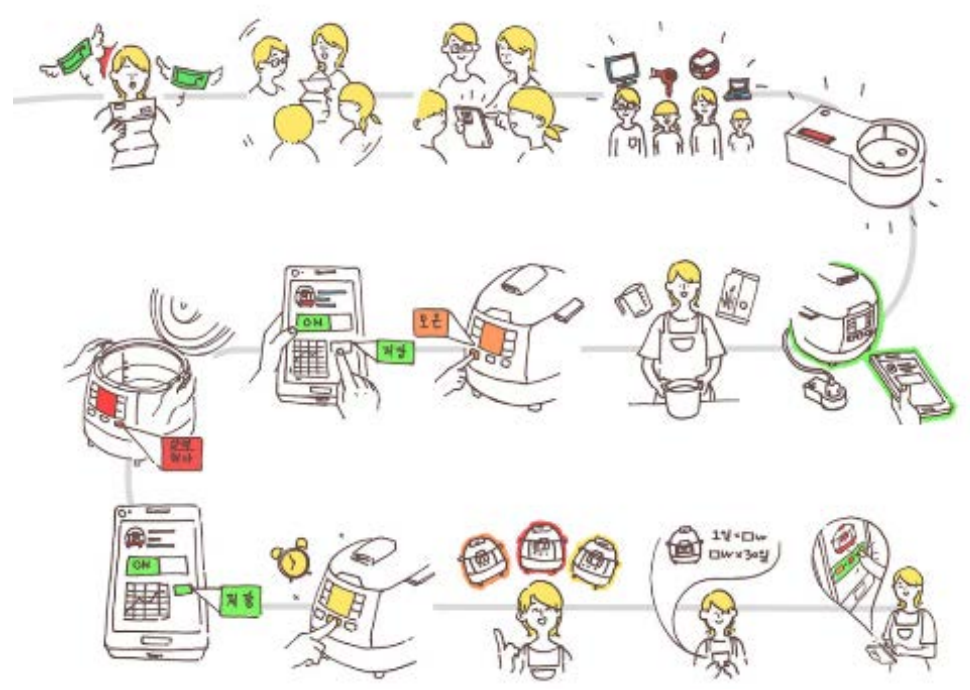

Figure 4. Service journey map of rice cooker usage with a smart plug.

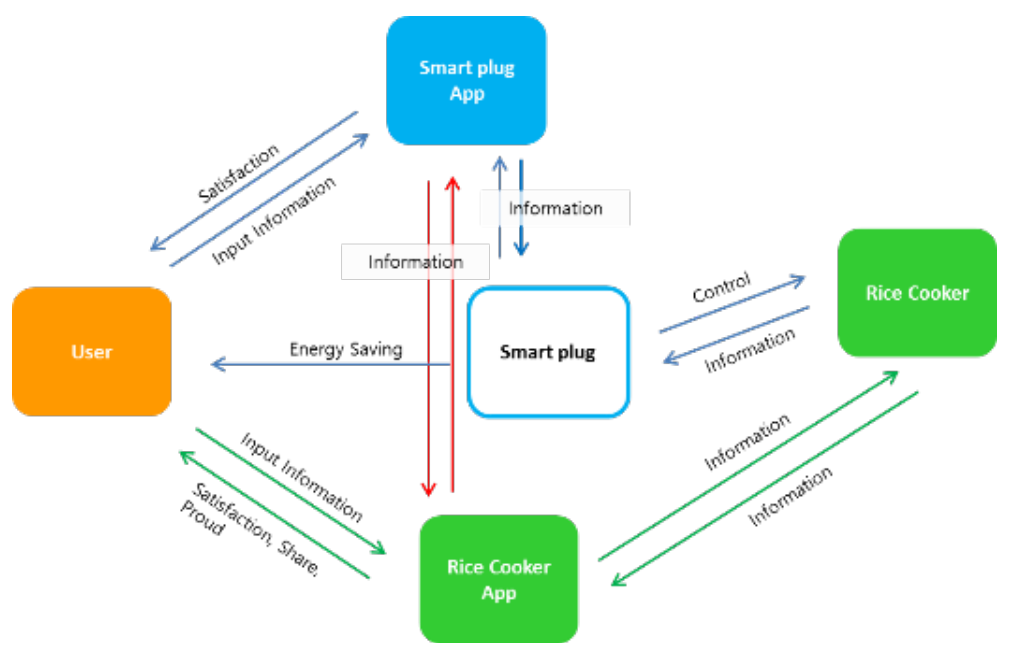

Figure 5. Service model of rice cooker usage with a smart plug.

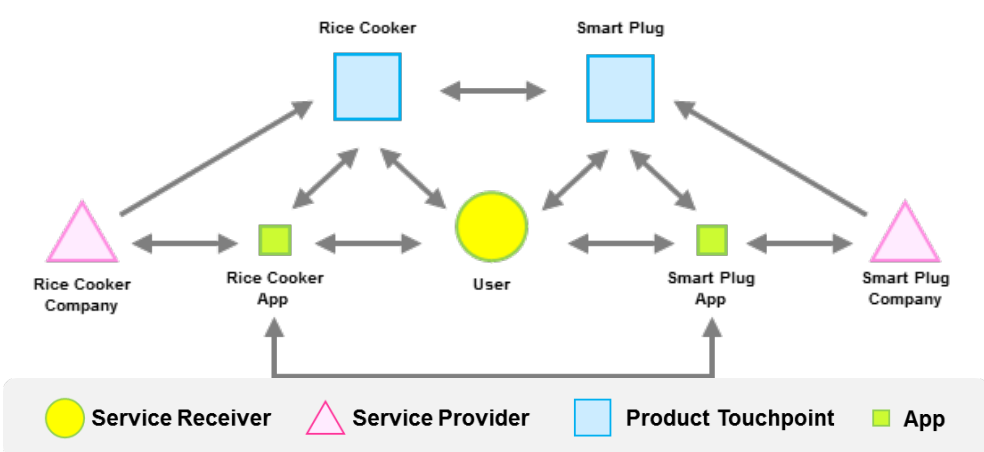

Figure 6. Social context representation of smart plug and rice cooker.

Attributed graph based systematic representation of social context of service interaction would not only help understanding of social interaction, but also support ideation process for PSS improvement. In Figure 8, the user node has the highest degree reflecting that the user is using the devices and is the most critical stakeholder. The 

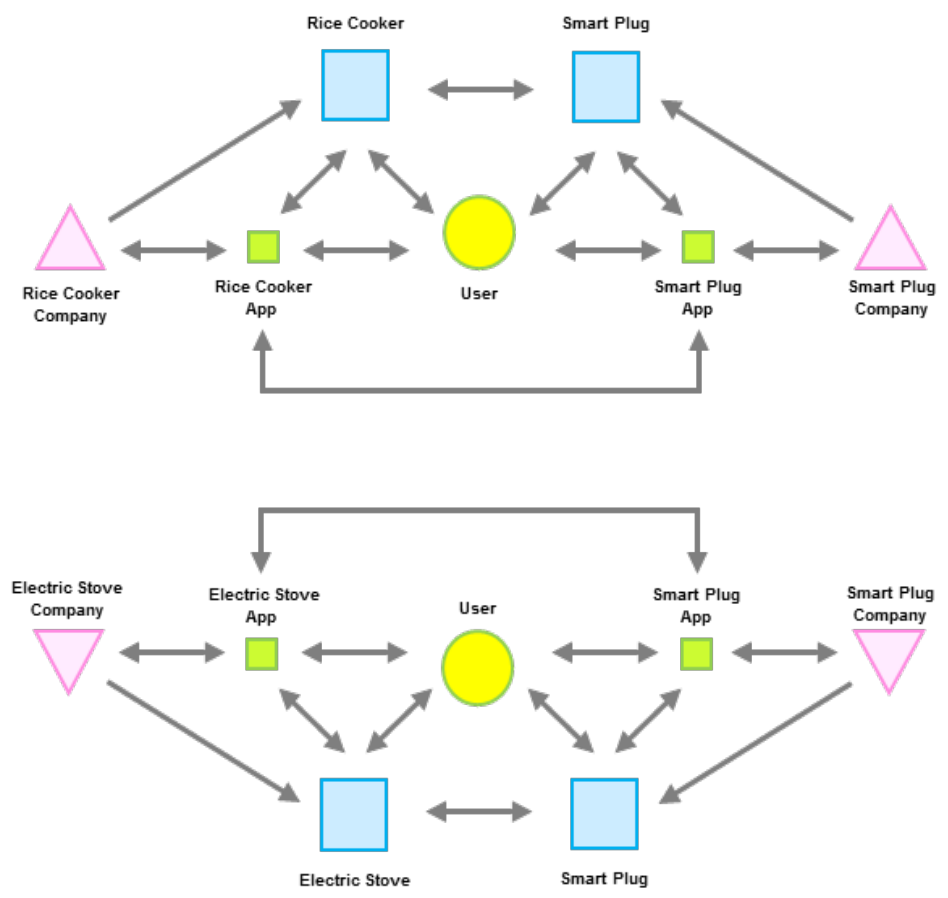

Figure 7. Social context representation of smart plug and electric devices.

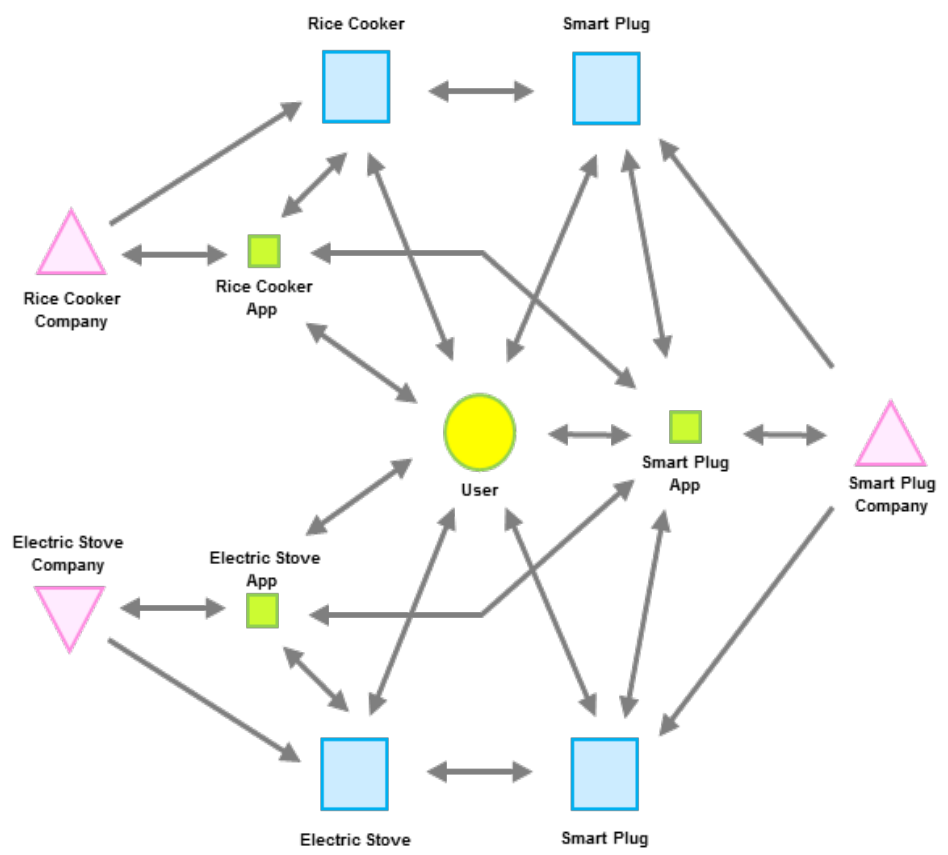

Figure 8. Social context representation of smart plug and electric stove.

node with the second highest degree is that of the smart plug app. From this observation, the smart plug company may establish its role to be a hub of IoT services of electric devices used at home. While the user is interacting with diverse electric devices in different ways reflecting the physical properties of different natures, user experience with the corresponding apps would pursue coherent experience in an integrated manner. Also social context representation can help classification of various PSSs so that their similarities and differences could be utilized in designing a new PSS. 


\section{Conclusion}

This paper has introduced a representation method for social interaction context of stakeholders and products in PSS. This paper has described the representation of social context of service interaction in PSS with IoT. As a specific case of PSS with IoT, Smart Plug PSS cases have been used to demonstrate the validity and utility of the representation. Based on the social contexts, the interaction among service providers, receivers and product touchpoints can be characterized. By having graph representations of social contexts, massively different PSS cases be compared effectively on their social inter-action aspects only. That is, while PSSs have many different issues, all these can be hidden except social interaction issues as represented by nodes and directed edges. In this projected space, comparisons are meaningfully done. This will allow that general improvements applicable on other PSS can be conceptualized for another PSS so that potential for improvement can be expanded. By defining interactions between them, researchers can create mutual benefit and contentment and find design improvement suggestion. As more cases will be designed and analyzed, the social context aspect of PSS concepts can be enriched by using such systematic representations. Our future work includes a PSS repository construction where many PSS cases are stored and their similarities and differences can be compared to help design improved PSS cases. Social interaction is an important dimension of the repository.

\section{Acknowledgements}

This work has been supported in part by the Korean Ministry of Trade, Industry and Energy, and in part by the Korean Ministry of Education.

\section{References}

[1] Kim, Y.S., Cho, C.K., Ko, Y.D. and Jee, H.S. (2011) E3 Value Concept for a New Design Paradigm. International Conference on Engineering Design (ICED), Copenhagen.

[2] Goedkoop, M.J., van Halen, C.J.G., te Riele, H.R.M. and Rommens, P.J.M. (1999) Product Service System, Ecological and Economic Basic. The Report No. 1999/36 Submitted to Ministerje van Volkshuisvesting, Ruimtelijke Ordening en Milieubeheer, Hague.

[3] Kim, Y.S. and Hong, Y.K. (2012) Interaction Model for Products and Services Using Affordance. Proceedings of ASME Computers and Information in Engineering Conference, Chicago. http://dx.doi.org/10.1115/detc2012-71110

[4] ISO 20282-1 (2006) Ease of Operation of Everyday Products, Part1: Design Requirements for Context of Use and User Characteristics. ISO.

[5] Kim, Y.S., Lee, S.W., Kim, J.H., Jeong, H., Lee, J.H. and Hong, Y.S. Product-Service Systems Design with Business Model Strategy Integrated: A Case Study of Urban Umbrella Rental Service System.

[6] Kim, Y.S., Do, S.H., Kim, J.H., Jeong, H., Lee, J.H. and Hong, Y.S. (2012) Product-Service Systems Design with Service Unit and Business Model Strategy: A Case Study of Make-Up Room Service. Design Engineering Workshop, Seoul.

[7] Belkins WEMO-SmartPlug. http://www.belkin.com/us/Products/home-automation/c/wemo-home-automation.

[8] Dawon, D.N.S. Power Manger-SmartPlug. http://www.powermanager.co.kr

[9] Lee, H.J., Kim, Y.S. and Park, S.H. (2014) Smart Plug Service Model and Business Models. Asia Design Engineering Workshop, Taipei.

[10] Donaldson, K., Ishii, K. and Sheppard, S. (2006) Customer Value Chain Analysis. Research in Engineering Design, 16. 